High Energy Phenomena in Relativistic Outflows III (HEPRO III)

International Journal of Modern Physics: Conference Series

Vol. 8 (2012) 225-230

(C) World Scientific Publishing Company

DOI: 10.1142/S2010194512004631

\title{
PHOTOSPHERIC THERMAL RADIATION FROM GRB COLLAPSAR JETS
}

\author{
AKIRA MIZUTA \\ Theory Center, Institute of Particle and Nuclear Studies, \\ KEK, 1-1 Oho, Tsukuba 305-0801, Japan \\ mizuta@post.kek.jp \\ SHIGEHIRO NAGATAKI \\ Yukawa Institute for Theoretical Physics, Kyoto University, \\ Kitashirakawa Oiwake-cho, Sakyo-ku, Kyoto, 606-8502, Japan \\ nagataki@yukawa.kyoto-u.ac.jp
}

\begin{abstract}
We have performed 2D relativistic hydrodynamic simulations of jets from a collapsar. The light curves and spectrum of the photospheric thermal radiation from ultrarelativistic gamma-ray burst (GRB) jets are calculated by the post process. At the head the jet has a dissipated region in which internal shocks can be seen and a freely expanding region follows. The freely expanding region shows a bullet like shape. The light curves continue about $100 \mathrm{~s}$ which corresponds to the jet injection duration. The light curves for $\theta_{v} \leq 2^{\circ}$ show quick rise and time variability for the first phase and low and steady luminosity phase follows. The light curves for $\theta_{v} \geq 3^{\circ}$ show slow rise and low luminosity. The spectrum below the peak energy is a power law and the index is $1 \sim 2.6$ which is softer than that of single temperature plank distribution Some of them are close to the observed one, i.e., Band function.
\end{abstract}

\section{Introduction}

The GRBs are one of the most energetic explosions in the Universe. The progenitors for at least some fraction of GRBs are massive stars. A gravitational collapse of the massive stars is an engine of the GRBs for such events. GRBs associated with supernova have been observed, for example, GRB030329/SN2003 dh ${ }^{1}$. Is is proposed that a relativistic jet which is launched from deep inside the star drills stellar envelopes and finally erupts the stellar surface, i.e., collapsar scenario ${ }^{2,3}$. During propagation of the jets in the interstellar medium, the prompt emission occurs, although the radiative mechanism of the prompt emission is not well understood. A non-thermal radiation, such as synchrotron radiation and/or inverse-Compton emission, can work for it. The thermal radiation from the photosphere also can be the radiation mechanism of the prompt emission ${ }^{4}$. In this paper we discuss on the photoshperic thermal radiation based on the numerical hydrodynamic simulations of the jet propagation from collapsar ${ }^{5,6,7}$. The light curves and spectrum of the thermal emission from the 
photosphere are calculated by a post process, considering the effect of the viewing angle.

\section{Methods, Computational Conditions, and Models}

The jet propagation from inside the progenitor to outside the progenitor is followed by the relativistic hydrodynamic simulations. We use 2 -D spherical coordinate $(r-\theta)$ for numerical hydrodynamic simulations, extending the computational box used in Mizuta et al. $(2011)^{7}$. The inner and outer computational boundary is $r_{\min }=10^{9} \mathrm{~cm}$ and $r_{\max }=1.2 \times 10^{13} \mathrm{~cm}$, respectively. The number of the radial grid is 3200 and spaced to logarithmic uniform with $\Delta r_{\min }=10^{7} \mathrm{~cm}$. The angular resolution from the polar axis $\left(\theta=0^{\circ}\right)$ to $30^{\circ}$ is $0.25^{\circ}$. The 60 uniform logarithmic grids are spaced in the range of $30^{\circ} \leq \theta \leq 90^{\circ}$. The code developed by one of the authors ${ }^{8,9}$ is used for the calculation.

The progenitor model 16TS which is developed by Woosley and Heger ${ }^{10}$ is employed. The progenitor has high spin and has about 16 solar masses at pre-SN. The radius of the progenitor is $4 \times 10^{10} \mathrm{~cm}$.

We inject the constant jet from inner computational boundary for the first 100 seconds. The power of the jet is $5 \times 10^{50} \mathrm{erg} \mathrm{s}^{-1}$, resulting the net released energy is $5 \times 10^{52}$ ergs in a jet. At this boundary the Lorentz factor $\left(\Gamma_{0}\right)$ is 5 and specific internal energy $\left(\epsilon_{0} / c^{2}\right)$ is 80 , where $c$ is speed of light. This jet parameter has a potential to be accelerated to Lorentz factor more than 500, if all thermal energy is converted to kinetic one without any dissipation. Two different initial opening angles of the jet are studied, i.e., $\theta_{0}=10^{\circ}$ and $\theta_{0}=5^{\circ}$. See also, Mizuta et al. $(2011)^{7}$ for more details on the numerical conditions.

The thermal radiation from the photosphere is calculated by the post process, using the results of the hydrodynamic simulations. We find photosphere at unity optical depth for the Thomson scattering. The optical depth $(\tau)$ is defined as follows;

$$
\tau=\int_{x_{\mathrm{ph}}}^{\infty} \sigma_{\mathrm{T}} \Gamma(1-\beta \cos \theta) n d l,
$$

where $\sigma_{\mathrm{T}}$ is the Thomson scattering cross section, $\Gamma\left(\equiv\left(1-\beta^{2}\right)^{-1 / 2}\right)$ is the Lorentz factor, $\beta$ is absolute value of velocity normalized by the speed of light, $\theta$ is the angle between the velocity vector $(\vec{\beta})$ and the line-of-sight (LOS), and $n$ is proper number density of the electron, $n \equiv 2 \rho / m_{\mathrm{He}}$, where $m_{\mathrm{He}}$ is the mass of helium atom. The expression in the integral includes the inverse of beaming factor $(\delta)$, i.e., $\Gamma(1-\beta \cos \theta)\left(\equiv \delta^{-1}(\theta)\right)$, to include the relativistic effect ${ }^{11}$. Here, we study the effect of the viewing angles, for an on-axis observer $\theta_{\mathrm{v}}=0$ and for off-axis observers, i.e., the angle between the jet axis and $\operatorname{LOS}, \theta_{\mathrm{v}}=1^{\circ}, 2^{\circ}, 3^{\circ}, 4^{\circ}$, and $5^{\circ}$.

Once the position of the photosphere is found, the isotropic luminosity of thermal radiation from the photosphere for the observers at infinity is evaluated as

$$
L_{\mathrm{iso}}\left(\theta_{\mathrm{v}}\right)=a c \int \delta(\theta)^{4} T^{4} \cos \theta_{\mathrm{ph}} d S
$$


where $a$ is the radiation constant, $\theta_{\mathrm{ph}}$ is the angle between the LOS and the normal vector of the emission surface ${ }^{12}$. The isotropic luminosity in the left hand side is considered by the observer time $\left(t_{\mathrm{obs}}\right)$ which is related with the laboratory time $\left(t_{\text {lab }}\right)$ as $t_{\mathrm{obs}}=t_{\mathrm{lab}}+d / c$, where $d$ is the distance between each photosphere and the observer. The integral is calculated as a summation of small areas and limited to a hemisphere to the observer. The local temperature at the photosphere is evaluated as $T=(3 p / a)^{1 / 4}$, where $p$ is the thermal pressure of the fluid.

\section{Results}

The jet shows successful eruption as shown in previous works, for example, Mizuta and Aloy $(2009)^{13}$. Before the eruption from the progenitor some internal shocks, such as oblique shocks, appear, due to the interaction between the jet and backflow $^{14}$. These internal structure remains after the eruption.

After the eruption from the progenitor, the jet proceeds to interstellar medium which is assumed to be low density. Figure 1 shows rest mass density and Lorentz factor contours at $t=20,165 \mathrm{~s}$, for the model with initial half opening angle 10 degrees. At the head of the jet, there is a little dissipated gas by the internal shocks. Then a freely expanding region follows. The freely expanding region grows up, as time goes on. It is quite tiny at $t=20 \mathrm{~s}$, only at $r<3 \times 10^{10} \mathrm{~cm}$, because the
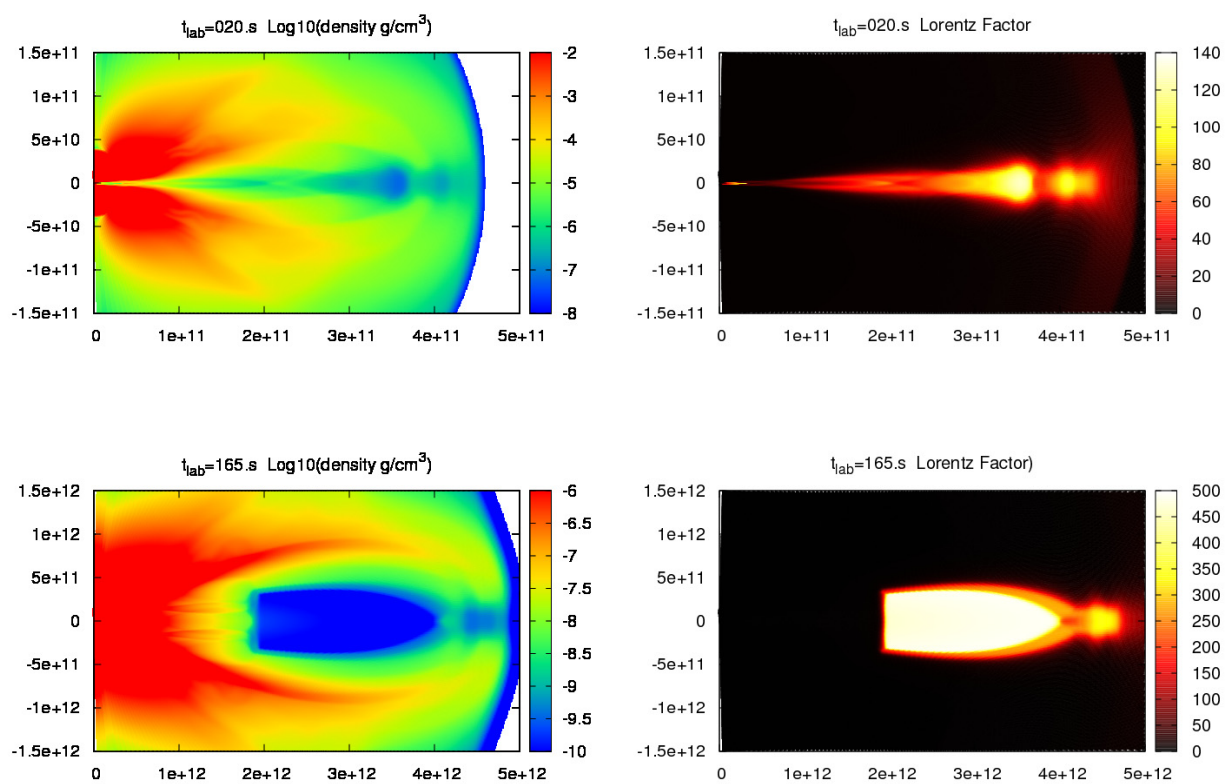

Fig. 1. Density and Lorentz factor contours of the model with initial half opening angle 10 degrees. 
expanding cocoon and dense expanding stellar envelopes surround the jet and work as a wall for the jet, resulting an appearance of the internal shocks. It takes some time for the jet to push away such walls. At $t=165 \mathrm{~s}$, a large freely expanding region at which the Lorentz factor is more than 400 can be seen. Since we turned off the injection at $t=100 \mathrm{~s}$, We can see a tail of the jet in the Lorentz factor contours. The Lorentz factor distribution in this freely expanding region seems a bullet. The model with the initial half opening angle 5 degrees show a little narrower structure and longer bullet structure than those of the model with the initial half opening angle 10 degrees.

The isotropic light curves for different viewing angle observers are shown in Fig. 2. It should be noted that the light curves are bolometric one, a fixed observing band the light curves would have different shapes than those shown here, see Mizuta et al. $(2011)^{7}$. The light curve for small viewing angle observers $\left(\theta_{\mathrm{v}} \leq 2^{\circ}\right)$ exhibits quick rise and very high luminosity phase for the first $30 \mathrm{~s}$. A few seconds time variability which is caused by the internal structure, such as density and Lorentz factor discontinuities can be seen. These properties are consistent with Lazzati et al. $(2011)^{5}$. At this phase the observer detects the radiation from the photosphere which is in the dissipated region at the head of the jet. Then the luminosity drops down about two orders of magnitude and shows steady feature. The observer detects the radiations from the photosphere which is in the freely expanding region. Since the velocity vector in this region is almost parallel to the radial coordinate and light cone is quite small due to large Lorentz factor (more than 400), only the components which is almost parallel to the LOS can be seen to the observer.

On the contrary the light curves for the large viewing angle observers $\left(\theta_{\mathrm{v}} \geq 3^{\circ}\right)$ exhibits low luminosity and flat properties for the first 10-20 seconds. Then the luminosity increases but the highest luminosities are not so high as that for small viewing angle observers $\left(\theta_{\mathrm{v}} \geq 2^{\circ}\right)$. In all models the light curves continue about 100 $\mathrm{s}$ It corresponds to the jet injection duration.
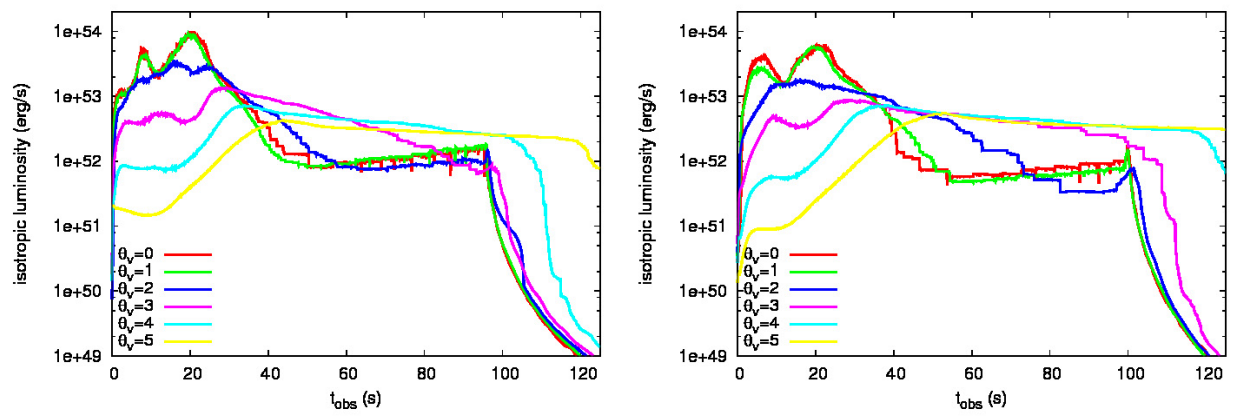

Fig. 2. The bolometric light curves for the observer at different viewing angles. The models with the initial half opening angle 10 degrees (left) and 5 degrees (right) are shown. fixed observing band the light curves would have different shapes than those shown here, see Mizuta et al.(2011). 
Figure 3 shows the spectrum for different viewing angle observers. Since the spectrum is constructed by the superposition of Planck distribution of different temperature, the spectrum is multi-colored. The case of the model with the initial half opening angle 10 degrees is shown. The $\nu F_{\nu}$ plots have a peak at a few tens keV to a few hundreds keV. The peak energy decreases, as the viewing angle increases. The spectrum index below the peak energy is about $2.4-2.5$ for $\theta_{\mathrm{v}}=0^{\circ}, 1^{\circ}, 2^{\circ}$. These indexes is harder than that of observed one, i.e., Band function ${ }^{15}$. On the contrary the spectrum index below the peak energy is almost unity for $\theta_{\mathrm{v}}=3^{\circ}, 4^{\circ}$ and $5^{\circ}$. The indexes are close to the observed one, though a Rayleigh Jeans tail at the range of a few keV can be seen.

\section{Conclusion}

We have performed long-term 2D relativistic hydrodynamic simulations of jets from a collapsar to see the photospheric thermal radiation. The light curves and spectrum of the photospheric thermal radiation from ultrarelativistic gamma-ray burst (GRB) jets are calculated by the post process. The jet is well collimated not only in the collapsar but also in the interstellar medium. At the head of the jet it has dissipated region in which there are internal shocks imprinted during the propagation in the progenitor. A freely expanding region which appears and grows up at the late phase follows. The freely expanding region shows a bullet like shape. The light curves continue about $100 \mathrm{~s}$ which corresponds to the jet injection duration. The light curves for $\theta_{v} \leq 2^{\circ}$ show quick rise and time variability for the first phase and low and steady luminosity phase follows. The light curves for $\theta_{v} \geq 3^{\circ}$ show slow rise

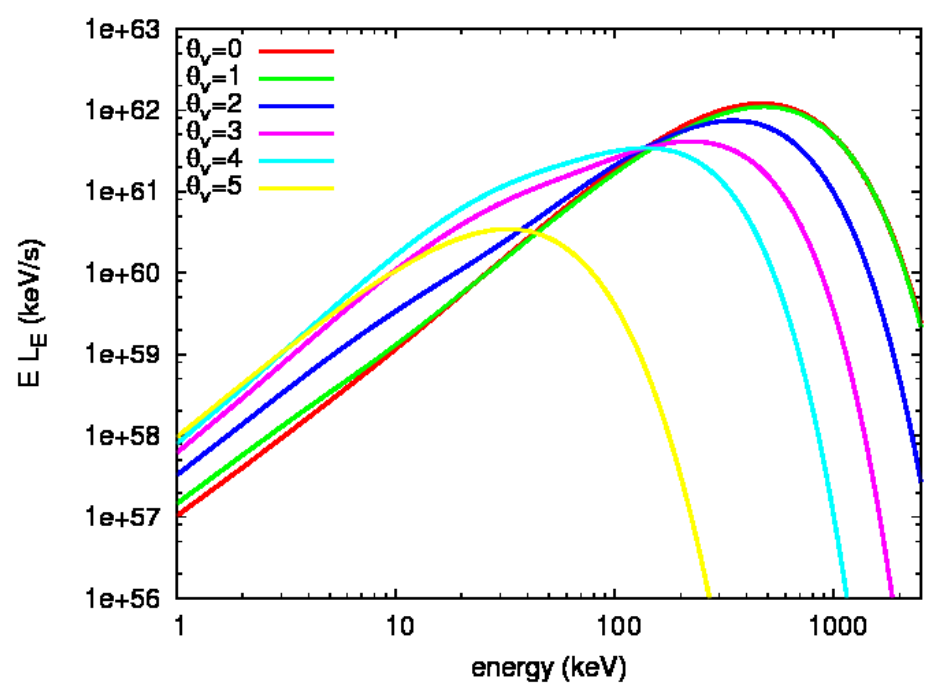

Fig. 3. The $\nu F_{\nu}$ plot for the models with the initial half opening angle 10 degrees. 
and low luminosity. The spectrum below the peak energy is a power law and the index is $1 \sim 2.6$ which is softer than that of single temperature plank distribution Some of them are close to the observed one, i.e., Band function.

\section{Acknowledgments}

We appreciate A. Heger for giving us their progenitor model data. This work is partly supported by Grants-in-Aid from the Ministry of Education, Culture, Sports, and Science and Technology (MEXT) (20105005 (A.M.) and 19047004, 21105509 (S.N.)), Japan Society for the Promotion of Science (JSPS) (19104006, 19740139, 21540304 S.N.), and a Grant-in-Aid for the Global COE Program "The Next Generation of Physics, Spun from Universality and Emergence" from MEXT of Japan. This work was carried out on NEC SX8, at YITP, Kyoto University, on the Space Science Simulator (NEC SX9) at JAXA, and on XT4 at CFCA at NAOJ.

\section{References}

1. K. Z. Stanek et al., Astrophys. J. Lett. 591, L17 (2003).

2. S. E. Woosley, Astrophys. J. 405, 273 (1993).

3. A. I. MacFadyen and S. E. Woosley, Astrophys. J. 262, 524 (1999).

4. M. Rees and P. Mészáros, Astrophys. J. 628, 847 (2005).

5. D. Lazzati, B. J. Morsony and M. C. Begelman, Astrophys. J. 73234 (2011).

6. H. Nagakura, H. Ito, K. Kiuchi and S. Yamada, Astrophys. J. 73180 (2011).

7. A. Mizuta, S. Nagataki and J. Aoi, Astrophys. J. 732, 26 (2011).

8. A. Mizuta, S. Yamada and H. Takabe, Astrophys. J. 606, 804 (2004).

9. A. Mizuta et al., Astrophys. J. 651, 690 (2006).

10. S. E. Woosley and A. Heger, Astrophys. J. 637, 914 (2006).

11. M. A. Abramowicz, I. D. Novikov and B. Paczynski, Astrophys. J. 369, 175 (1991).

12. A. Pe'er et al., Astrophys. J. Lett. 664, L1 (2007).

13. A. Mizuta and M. A. Aloy, Astrophys. J. 699, 1261 (2009).

14. A. Mizuta, M. Kino and H. Nagakura, Astrophys. J. Lett. 709, L83 (2010).

15. D. Band et al., Astrophys. J. 413, 281 (1993). 